\title{
EMPLEO FEMENINO Y MEDIOS DE COMUNICACIÓN: DIRIGIR EN FEMENINO
}

\author{
María Pilar Rodríguez \\ Universidad de Deusto \\ mpirodrig@gmail.com
}

Recibido: 23-01-09

Aceptado: 02-03-09

\section{Resumen}

El presente artículo refleja los resultados del estudio desarrollado por el equipo de investigación Género y medios de comunicación de los departamentos de Comunicación y Empresa de la Universidad de Deusto, y publicado por la Editorial LID bajo el título Dirigir en femenino. El libro aborda el tema del mundo del trabajo por parte de las mujeres desde una triple perspectiva. Por un lado se retrata la realidad laboral de las mujeres en España a través de datos secundarios. En segundo lugar se realiza un vaciado de noticias de prensa y se analiza tanto la prensa diaria general y económica como las publicaciones dirigidas a público femenino para ver el tratamiento que los medios de comunicación hacen de las mujeres en ámbitos empresariales. En tercer lugar se realiza una encuesta a una muestra representativa de la CAPV que pretende conocer la percepción del público en general de la realidad de las mujeres directivas tal y como la conocen a través de los medios. Todo ello se completa con la aportación de algunas protagonistas de los medios de comunicación y del mundo empresarial.

Palabras clave: Empleo, género, medios de comunicación, techo de cristal, percepciones sociales. 


\begin{abstract}
This article presents the results of a research project carried out by the Gender and Media research group at Deusto University in San Sebastián, and published under the title Dirigir en femenino by LID. The book analyzes work and labour practices as related to women from a triple perspective. Firstly it shows a picture of the situation in Spain through data and reports focused on various aspects such as female unemployment, female leadership or the glass ceiling, among others. Then, it studies Spanish economic and daily press and shows the results of a detailed analysis on the portrayal of business women. Finally, the results of an opinion survey carried out in the Basque Country provide an accurate image of the social perceptions around business women and their media projections. To conclude some influential business women give their own views on the matter.
\end{abstract}

Key words: Work, gender, media, glass ceiling, social perceptions, business women.

\title{
1. Introducción
}

El presente artículo refleja los resultados del estudio desarrollado por el equipo de investigación Género y medios de comunicación de los departamentos de Comunicación y Empresa de la Universidad de Deusto - San Sebastián, dirigido por la autora de este artículo y formado por María Asunción Ibáñez, María Jesús Korkostegi, Lorea Narbaiza, María Jesús Pando y Begoña Sanz. El libro, titulado Dirigir en femenino, traza en su primer capítulo el panorama laboral de las mujeres en el Estado español. La situación laboral de las mujeres en España presenta un panorama que muestra particularidades del mercado de trabajo que todavía hoy establecen claras diferencias entre los géneros. Si bien la situación de igualdad en cuanto a las oportunidades de empleo y en cuanto a las retribuciones salariales no se ha logrado, es cierto que la exploración del futuro hace albergar esperanzas reales de avances significativos. Actualmente la participación de las mujeres en el mercado laboral 
aumenta, y crece también el número de mujeres empleadas con estudios superiores, con lo que su presencia se hace patente en gran parte de los sectores económicos. Sin embargo, como señala la introducción del informe especial Mujer y empresa elaborado por Tecnociencia: «si bien es cierto que las mujeres acceden al trabajo, no deja de ser menos cierto que aún se sitúan lejos del poder, feudo que aún mantiene la población masculina. La tasa de incorporación de la mujer a cargos directivos es aún menor al $5 \%{ }^{1} »$. Este mismo informe recoge los resultados de la Encuesta de Población Activa 2005 (EPA), realizada por el Instituto Nacional de Estadística, cuyas principales aportaciones se indican a continuación. La tasa de actividad en España en el mercado de trabajo femenino alcanza en el período que abarca desde 1982 hasta 2004 el 45,09\% del total. Estas cifras se han incrementado en un $15 \%$ en los últimos veinte años. Pese a este hecho, la diferencia con respecto a la tasa de actividad masculina es muy elevada, ya que ésta se sitúa en torno al $67,72 \%$ del total de hombres, lo que supone una importante brecha entre ambos sexos. Si se comparan estas tasas de actividad con el resto de la UE se observa que la tasa de actividad femenina en España es inferior a la media del total de los 15 países miembros tradicionales (tan sólo Grecia e Italia poseen una tasa inferior a la española). En lo referente a la composición del mercado de trabajo femenino, éstos son los datos que proporciona el mencionado informe en el apartado El mercado laboral en cifras.

"En cuanto a la composición del mercado de trabajo femenino, cabe destacar que las profesiones que registran una tasa de ocupación mayor son las de administrativo y aquéllas relacionadas con servicios de restauración, personales, protección y de venta. A continuación se encuentran las técnicas profesionales científicas e intelectuales, que registran una tasa de ocupación muy similar a aquellas mujeres que no precisan cualificación para realizar su

\footnotetext{
${ }^{1}$ TECNOCIENCIA. Especial Mujer y Empresa.

http://www.tecnociencia.es/especiales/mujer_empresa/introducción.htm
} 
trabajo. La tasa de ocupación inferior es la registrada por los trabajos de corte artesano, de industrias manufactureras, construcción y minería”.

Si bien en ciertos aspectos estos datos cumplen las expectativas previstas, se aprecia una tendencia a la inclusión mayoritaria de mujeres en los puestos técnicos profesionales de tipo científico e intelectual, lo que hace albergar esperanzas para el futuro profesional del mercado laboral femenino. Por último, la $\mathrm{EPA}^{2}$ también refleja el indicador de la tasa de paro femenino, que se sitúa en la actualidad en torno al 14,39\%. Si bien a comienzos de los años noventa el indicador muestra una tasa que supone el doble de paro femenino que en el momento actual, hay que destacar que incluso hoy en día la tasa de paro femenino duplica a la tasa masculina, situada en torno al 7,55\%. El Informe recoge como dato más significativo el hecho alarmante de que la tasa de paro de las mujeres españolas duplica la media de todas las tasas de los principales países miembros, lo que refleja un problema estructural de la propia composición y funcionamiento del mercado de trabajo en lo que a la población femenina se refiere, sobre todo si se tiene en cuenta que las cifras de paro masculinas están en equilibrio con la media europea. En el apartado dedicado a la discriminación salarial, el informe mencionado, elaborado por Tecnociencia, se basa en el estudio titulado Encuesta de Estructura Salarial 2002, elaborado por el Instituto Nacional de Estadística, realizado de forma armonizada en toda la Unión Europea, y advierte que los datos se refieren al año 2002, pero añade que ni la composición ni las diferencias salariales han experimentado grandes cambios en su evolución. Éstos son los resultados: el salario bruto anual medio en 2002 fue de 22.169,16 euros en el caso de los varones y de 15.767,56 euros para las mujeres trabajadoras. Esto supone que el salario promedio

\footnotetext{
${ }^{2}$ Importa constatar que en el informe correspondiente al cuarto trimestre de 2008, la Encuesta de Población Activa arroja cambios significativos con respecto a los datos recogidos en el libro: por encima de los 10 millones (10.035.200). La tasa de actividad femenina aumenta 92 centésimas, hasta alcanzar el 51,38\%. Por su parte, el número de activos varones se cifra en 13.029.500, con un descenso de 83.000. La tasa de actividad masculina cae seis décimas en el último trimestre del año y queda en el $69,21 \%$. En los últimos 12 meses el número de activos ha aumentado en 660.200, de los que 507.100 son mujeres.
} 
anual femenino es el 71,1\% del masculino. Por ramas de actividad, Mujer y empresa señala que el análisis de las diferencias de salario entre hombres y mujeres para las distintas actividades económicas muestra que en todas ellas los salarios femeninos son inferiores. En cuanto al estudio comparativo de salarios y ocupación, éste también arroja los mismos resultados, y permite concluir que en todas las ocupaciones las mujeres poseen un salario inferior al de los hombres.

Las cifras referentes al número de mujeres en puestos directivos varían en función del año de elaboración de los estudios y del modo de obtención de los porcentajes. De acuerdo con los resultados del estudio elaborado por la consultora de recursos humanos Hudson en 2007, el número de mujeres contratadas para ocupar puestos de dirección a nivel nacional asciende hasta el 24,9\%. El estudio se llevó a cabo sobre una muestra de 17.300 personas que ocupan puestos de dirección, de las cuales sólo 3.944 son mujeres. El estudio revela que las áreas donde las mujeres pueden equipararse a los hombres son las de recursos humanos, atención al cliente, marketing o calidad. El análisis también constata la dificultad de las mujeres para acceder a los puestos más altos, tales como los Consejos de Dirección o de Presidencia. No es posible hablar de una única razón como la causa de esta situación. Por un lado, si bien las mujeres ya tienen una presencia mayoritaria en las universidades, es necesario esperar algún tiempo para que esas mujeres que despuntan en la universidad vayan accediendo a los puestos directivos. Pero más significativo sigue siendo el gran obstáculo que para muchas mujeres todavía hoy constituye la conciliación de la vida laboral con la vida familiar, asunto que se explorará con detalle más adelante. Por último, y en algunas ocasiones que cada vez son menos frecuentes, existen reticencias para la contratación de mujeres para esos puestos de mayor responsabilidad y poder. Los hombres ocupan hoy en día el $97 \%$ de los puestos directivos de las empresas del Ibex 35 y cobran un 30\% más que las mujeres, según los datos del Instituto Nacional de Estadística y del Instituto de la Mujer. En efecto, si la presencia femenina en la alta dirección sigue siendo escasa, en los consejos de administración pasa a ser meramente testimonial. Los datos obtenidos en el estudio Diversidad de género en los consejos de administración de las 
sociedades cotizadas y cajas de ahorros españolas no difieren de los presentados en investigaciones similares: «Así, el estudio evidencia que la diversidad de género en los consejos de administración de las sociedades cotizadas españolas es reducida, pues tan sólo el $4 \%$ de los asientos de los consejos eran ocupados por mujeres a finales del año 2004». Además, la mayoría de estas mujeres (un 70\%) son nombradas como consejeras y ejecutivas dominicales. La presencia de mujeres en cargos de Presidencia, Vicepresidencia y puesto de Secretario de los consejos es incluso más reducida y no llega al 3\% de las sociedades. En puestos de alta dirección se alcanza un 4,5\%. Este estudio apunta otro dato reseñable, y es que la representación de las mujeres en los consejos de administración de las sociedades cotizadas españolas varía en función del sector de actividad al que pertenezcan las compañías, puesto que sectores como el de bienes de consumo, servicios de consumo y servicios financieros e inmobiliarios presentan una representación porcentual superior a la media, mientras que los de materiales básicos, de tecnología y comunicaciones y de petróleo y energía tienen una representación menor de mujeres que el promedio total. Los datos obtenidos sitúan a los consejos de administración de las sociedades cotizadas españolas en las últimas posiciones del contraste entre los países europeos en cuanto a diversidad de género se refiere. La edición digital de El País ${ }^{3}$ con fecha del 21 de enero de 2007 informaba acerca de la entrada en vigor del Código Unificado de Buen Gobierno, de modo que las cincuenta y siete recomendaciones aprobadas por el grupo de trabajo presidido por Manuel Conthe se han ido aplicando de modo que las empresas puedan rendir cuentas de sus prácticas de gobierno corporativo. En el momento de aplicación del Código Conthe ninguna empresa se adaptaba a esos nuevos estándares, cuyo cumplimiento en todo caso es voluntario. En el caso de las mujeres, el código no fija un número determinado, pero anima a aumentar su presencia. Urge que estas recomendaciones se tomen en cuenta y que las empresas apliquen con responsabilidad los códigos

\footnotetext{
${ }^{3}$ http://www.elpais.com/articulo/economia/empresa/cumple/Codigo/Conthe/elpepueco/20070121e lpepieco_1/Tes
} 
encaminados a favorecer la presencia de las mujeres en los consejos y en otros puestos de dirección.

\section{El techo de cristal y el liderazgo femenino}

Hay numerosos factores que se alían para explicar esta dificultad persistente de acceso a los niveles más altos de decisión y de poder en las empresas. En primer lugar hay que hacer referencia al sistema utilizado para nombrar a los consejeros, ya que en muchas ocasiones ocurre que en la práctica es el presidente quien designa o propone a los futuros consejeros dentro de un círculo cerrado de candidatos que casi nunca son mujeres, en lugar de existir un sistema establecido liderado por una comisión de nombramientos en función del perfil que necesita la compañía en cada momento. Según explica Carlos Alemany, director general de Korn Ferry, hay otras causas que no deben ser desdeñadas, tales como la escasa visibilidad de muchas y muy buenas profesionales, pero también en ocasiones, la falta de preparación o formación específica para formar parte de los consejos de administración: «No por ser un buen ejecutivo se está preparado para ser consejero y es cierto que son más numerosos los hombres que cuentan con la preparación jurídica para conseguirlo» ${ }^{4}$. Con todo, parece que en los últimos meses del año 2007 se han ido produciendo algunos cambios alentadores que indican que existe un ligero incremento de las mujeres precisamente en los consejos de las grandes empresas españolas. Así, por ejemplo, la noticia recogida en Expansión \& Empleo con fecha 29 de septiembre de 2007 nos dice que el 6\% de los puestos externos de los consejos de administración están hoy en día ocupados por mujeres, superando el $4 \%$ anterior. Las razones que se aducen como causas de este incremento (que sin ser espectacular sí

\footnotetext{
${ }^{2}$ Citado en «¿Dónde están las mujeres directivas?», en Expansión \& Empleo, 30 de junio y 1 de julio de 2007, pág. 3.
} 
resulta significativo) son precisamente las derivadas de la aplicación de la Ley de Igualdad por una parte y, por otra, las derivadas del hecho de que son las hijas las que poco a poco comienzan a ocupar los puestos de responsabilidad en las empresas familiares.

Como señala Juan San Andrés en su artículo «El techo de cristal resiste», hace algo más de veinte años que se acuñó esa expresión, proveniente de la inglesa the glass ceiling, para referirse a la barrera imperceptible que permite a las mujeres avistar las cimas de la carrera profesional en las corporaciones pero no alcanzarlas, y afirma que es racionalmente incomprensible que las empresas convivan con el desperdicio de capacidad que supone la existencia del techo de cristal. En Estados Unidos varios estudios han demostrado que existe un alto porcentaje de mujeres que tienen las más altas calificaciones académicas y que sin embargo abandonan brillantes carreras. Propone el autor que las mujeres irían perdiendo interés por subir peldaños a sabiendas de que una vez arriba terminarán por frenar su ascenso, y ello actuaría como el detonante de un círculo vicioso en el que al ver la mujer que es poco considerada para puestos de dirección, pierda interés, sea menos reconocida y dé la razón a quienes les ignoran por lo que sus posibilidades disminuyen. Termina el autor advirtiendo de la gravedad de la situación y llamando la atención a los consejeros, a los que pregunta: « ¿No les llama la atención que haya tan pocas directivas? ¿No les preocupa el derroche de talento que las prácticas de segregación silenciosa siguen produciendo?». 5 María Teresa Fernández de la Vega, en su intervención en calidad de vicepresidenta del Gobierno en la presentación del informe Diversidad de género en los consejos de administración de las sociedades cotizadas y cajas de ahorros españolas, caracterizaba de este modo los orígenes y las razones de continuidad del fenómeno que se analiza:

\footnotetext{
${ }^{3}$ Juan San Andrés. «El techo de cristal resiste», en Expansión y empleo.com (28 de enero de 2006).

http://www.expansionyempleo.com/edicion/expansionyempleo/opinion/es/desarrollo/970400.html
} 
"La cultura patriarcal ha impregnado el mundo profesional y empresarial. En él las mujeres han sido generalmente invisibles y su actividad, frecuentemente, considerada de una categoría inferior. Las barreras ocultas, como la familia, la falta de asesoramiento y de modelos femeninos, las actitudes abiertamente sexistas, el escaso control de las redes informales de influencia, siguen fortaleciendo el denominado techo de cristal que impide el progreso profesional de las mujeres. Y así es: cuanto más alto es el rango, el prestigio y el poder, menor es la proporción de mujeres".

En el estudio titulado Mujeres Directivas: Promoción profesional en España y el Reino Unido, las autoras constatan que uno de los factores que continúa teniendo un enorme peso en la obstaculización del progreso profesional de las mujeres es la cultura organizacional basada en creencias estereotipadas de género, que afectan - de modo difícil de detectar - a las políticas de selección de personal y a las escasas posibilidades de promoción femenina a los cargos directivos. El estudio Rompiendo el techo de cristal propone las siguientes medidas de acción encaminadas al mismo propósito: en primer lugar, el fomento de la coeducación en valores inclusivos en los que hombres y mujeres compartan un espacio común de modo que se favorezca el intercambio de roles de género productivo y reproductivo. En segundo lugar inciden en algo reseñado en todos los estudios mencionados: consideran necesario adoptar medidas de flexibilización del trabajo, políticas de selección y promoción no discriminatorias, políticas salariales que tengan en cuenta los resultados, sistemas de evaluación globales y medidas para la conciliación de la vida familiar y laboral.

Con respecto a la conciliación de vida familiar y laboral, y como señala el informe elaborado por Tecnociencia en el apartado titulado «El trabajo doméstico», diez millones de amas de casa españolas están consideradas oficialmente como población inactiva. De acuerdo con la Encuesta de Población Activa del Instituto Nacional de Estadística, las amas de casa son mujeres en el 98\% de los casos, y la visión del trabajo doméstico como una actividad que las mujeres deben llevar a cabo está arraigada en la 
ideología social hasta considerarse que naturalmente ha de ser así. Los datos que reflejan la dedicación de las mujeres a las tareas domésticas o los permisos de maternidad frente a los de paternidad demuestran a las claras la excesiva desproporción que existe en todo lo que atañe a las tareas domésticas y al cuidado de los niños y otros familiares dependientes. Como consecuencia de la proclamación de la Ley de Igualdad y de su implantación en las empresas las ejecutivas y empresarias se muestran mayoritariamente más proclives a buscar y aplicar políticas y hábitos que favorezcan la conciliación de la vida familiar y laboral que a la aprobación de leyes en términos de cuotas paritarias. Tales políticas insisten en la necesidad de compartir las tareas y las responsabilidades familiares entre mujeres y hombres, ya que en todos los estudios realizados hasta la fecha se muestra un desequilibrio acentuado entre el número de horas que cada sexo dedica diariamente a las tareas del hogar y al cuidado de las personas dependientes, tanto si la mujer tiene un empleo remunerado fuera del hogar como si no lo tiene, y si uno de los miembros decide abandonar temporalmente el mercado laboral para dedicarse al cuidado de los hijos, es la mujer en un 96,38\% quien lo hace. Estas decisiones van más allá de esas abrumadoras cifras y tienen consecuencias a largo plazo, ya que cuando las mujeres deciden reincorporarse al mercado laboral se encuentran con dificultades como la edad, la falta de preparación o la incompatibilidad con las obligaciones familiares que se han acostumbrado a solucionar ellas solas.

En lo tocante al liderazgo femenino, hay numerosas apreciaciones que han aflorado recientemente en lo tocante a los diferentes estilos de dirección de hombres y mujeres, y en general se tiende a percibir que las mujeres ponen en práctica un estilo de economía más humana y más eficaz. La conclusión que ofrece Ester Barberá en su trabajo titulado Rompiendo el techo de cristal: los beneficios de la diversidad de género en los equipos de dirección subraya que las mujeres, debido a los procesos de socialización de género que experimentan en sus vidas cotidianas, aportan una serie de valores, actitudes y competencias a sus puestos de trabajo y poseen un estilo de dirección muy valorado en la actualidad, que se traduce en una mejora en el rendimiento, en el clima y en la satisfacción laboral. Sin entrar en consideraciones de 
diferenciación esencial biológica o de carácter que puedan marcar la escisión entre los modos de actuar de hombres y mujeres, sí interesa atender a las ventajas que las particularidades del liderazgo femenino presentan para el mundo empresarial. El capítulo concluye con algunas reflexiones en torno a la Ley de Igualdad, su incidencia en la corrección del desequilibrio entre hombres y mujeres en el acceso al mercado de trabajo y, en particular, en la incorporación de las mujeres a los puestos más altos de poder y responsabilidad.

\section{La prensa escrita: análisis cualitativo y cuantitativo}

Tomando como referencia la situación descrita en el capítulo anterior, los capítulos dos, tres y cuatro se centran en el análisis de la prensa escrita. Este estudio de la presencia de las mujeres en el ámbito laboral se hace a partir del convencimiento de que los medios de comunicación no solucionan los conflictos o problemas, pero sí pueden generar un ambiente propicio en la sociedad para promover ciertas mejoras. Sin duda, la investigación acerca de los logros y carencias de los medios de comunicación en torno a la imagen que proporcionan de las mujeres en el ámbito empresarial puede ayudar a una correcta visualización de su experiencia y provocar una concienciación social que contribuya a la igualdad de oportunidades de hombres y mujeres en las empresas. El estudio de los medios de comunicación y el análisis de sus logros y carencias en la imagen que proporcionan de las mujeres en el ámbito empresarial ayudará, sin duda, a una correcta visualización de su realidad y ello, a su vez, traerá consigo una concienciación social que permita la igualdad de oportunidades de hombres y mujeres en las empresas. Por ello se han seleccionado seis publicaciones: cuatro diarios de información general y dos diarios económicos. Los diarios de información general elegidos han sido dos de tirada nacional (El Mundo y El País) y dos del País Vasco (El Diario Vasco y Gara). En cuanto a los económicos, el análisis se centra en Expansión y Cinco Días. 
Tras un detallado análisis cuantitativo y cualitativo, se advierte que la ideología del medio escrito y el perfil de público al que va dirigido determinan no sólo la selección del tipo de noticias, sino también el tratamiento que se les da. Así, en el contexto general de mujeres y empresa, las seis publicaciones no siempre tratan los mismos aspectos de esta compleja realidad y, además, cuando abordan un mismo tema no lo hacen con la misma intensidad. La prensa económica, cuyos destinatarios son los/as expertos/as en temas de empresa y economía, obvia los temas relacionados con los conflictos laborales, con los colectivos más desfavorecidos y, por lo mismo, las voces sindicales están ausentes. La generalista, a su vez, por estar abierta a un sector más amplio de la población, está obligada a contemplar todas las vertientes de esa realidad, pero, dependiendo de la línea editorial del diario, las preferencias por unas u otras temáticas se hacen palpables. Lo anteriormente expuesto conduce irremediablemente a cuestionar el tratamiento informativo de la prensa en general, ya que presenta de manera fragmentada la situación de las mujeres en la empresa. Priman en ocasiones los intereses del medio y no su responsabilidad de dar cuenta de toda la realidad en su complejidad.

Para concluir, podemos preguntarnos adónde conducen las anteriores reflexiones. Indudablemente, a hacer conscientes a los medios escritos de la repercusión social que tiene lo que comunican y cómo lo hacen; algo que, hoy por hoy, resulta de total necesidad. No en vano afirma Teun Van Dijk que los medios cuando tratan la información realizan actos comunicativos con intención y contribuyen a la interpretación de la realidad al igual que otras instituciones, por lo que son responsables del mensaje que reciben sus lectores/as. Responsables, si no los únicos, de los cambios de la sociedad, deben tomar como pauta del buen trabajo periodístico, en lo que concierne al tema que nos ocupa, el no caer en la rutina y ser coherentes con aquellos principios con que se estrenan en el mercado cuando inician su andadura. Se ofrecen además algunas recomendaciones para mejorar los modos de tratar este tema en la prensa en general, para que los frutos sociales de la igualdad entre hombres y mujeres en el trabajo se hagan realidad por el bien de la propia sociedad. 
El tercer capítulo se centra en un acercamiento crítico a la prensa escrita y presta especial atención al enfoque de la noticia. Todas las noticias se construyen desde una posición ideológica y un espacio social, desde una línea editorial, desde unos condicionantes que no solo definen la naturaleza de la información sino que también dibujan el contorno tras el que se excluyen una multitud de informaciones que nunca llegarán a formar parte de la realidad informativa y, por tanto, difícilmente llegarán a conocerse y formar parte de nuestras realidades. A través de esta mirada tomamos conciencia, como público lector, de la importancia que tiene el tratamiento informativo de la realidad, no solo lo que forma esa actualidad informativa, sino también - y sobre todo en muchos casos - lo que se calla, lo que se obvia, se ignora o, simplemente, nunca llega a un teletipo. Si en el capitulo anterior aparecía un panorama en cifras centrado en la incidencia de noticias referidas a mujeres en ámbitos empresariales en los medios de comunicación escritos, en este capítulo se analizan de forma más detenida ciertas noticias vinculadas temáticamente entre ellas, para desbrozar los mecanismos que operan en la presentación de la noticia.

El análisis crítico de las noticias de mujeres en el ámbito empresarial lleva, por una parte pero no exclusivamente, al estudio cuantitativo y cualitativo de las figuras femeninas que aparecen como protagonistas de las noticias y va más allá de las noticias referentes a mujeres particulares o grupos de mujeres. También se ocupa de informaciones sobre asuntos que directamente afectan la presencia y el futuro de las mujeres en la empresa, como puede ser la Ley de Igualdad, la política de cuotas, los conflictos laborales o la conciliación laboral. Las unidades de análisis las configuran los titulares, la ubicación de la noticia, el género informativo o interpretativo, las protagonistas (tanto individuales como colectivos), y el propio texto (qué información se proporciona y cómo). Estas unidades se utilizan para abordar el análisis de contenido de prensa de interés general y prensa económica pero también para abordar el análisis contrastivo de algunos temas como la Ley de Igualdad o la conciliación familia-trabajo, que han tenido una especial presencia en prensa en el periodo de análisis llevado a cabo. El capítulo concluye con la afirmación de que las mujeres siguen sin participar 
plenamente de los espacios del núcleo duro informativo, de las hard news: su presencia en portada, contraportada y portada de sección son una buena muestra de dónde están y lo que les queda por recorrer. De momento los logros se vislumbran en espacios informativos pequeños, modestos, llegando incluso a ocupar alguna página central de importancia. No se dispone de datos que permitan contrastar este fenómeno hace tres, cinco, diez años, pero nos atreveríamos a afirmar que su presencia en los medios económicos entonces era nula. Se constata, pues, un avance significativo en su presencia cuantitativa y cualitativa en prensa.

El estudio de prensa generalista y económica se complementa en el capítulo cuatro con el análisis de Yo Dona y Mujer Hoy. Su perfil editorial - publicaciones femeninas de periodicidad semanal distribuidas a través de diarios - nos permite indagar si en las páginas de este tipo de suplementos aparecen y en qué proporción, las actividades llevadas a cabo por las mujeres empresarias y directivas y los asuntos que les conciernen directamente en su desarrollo profesional; en qué secciones se recogen y de qué manera se abordan, es decir, qué mensaje se transmite. El público objetivo de ambas publicaciones, las mujeres, hace pensar en la posibilidad de una mayor aproximación de Yo Dona y Mujer Hoy a la realidad profesional de las directivas y las empresarias. Sin embargo, las tradicionales agendas temáticas de estas revistas enfocadas en la moda, la decoración, la belleza, el ocio y la actualidad, suscita la duda de si tiene cabida en ellas también ese asunto. Los resultados obtenidos en el análisis de contenido de los 26 ejemplares editados entre junio y diciembre del 2006 por cada una de las publicaciones ofrecen una respuesta positiva al respecto, sobre todo en lo referido a la más reciente, Yo Dona, que irrumpe en el mercado editorial en mayo del 2005 Mujer Hoy existe desde 1999. La revista logra conjugar la promoción e información de productos de última tendencia y de sus grandes firmas con la presentación de mujeres cuyas semblanzas ponen de relieve la creciente presencia femenina en cargos empresariales importantes. 


\section{Percepción social: encuestas en la Comunidad Autónoma del País Vasco}

En el capítulo cinco se dan a conocer los resultados de la encuesta que realizamos durante el mes de febrero de 2007 para conocer la percepción que la opinión pública tiene acerca de la realidad de las mujeres empresarias y directivas y sobre su reflejo en los medios informativos impresos. El objetivo de este estudio empírico es conocer cuál es la percepción que tiene la población de la Comunidad Autónoma del País Vasco (en adelante CAPV) de la realidad de las mujeres empresarias y directivas, y qué opina sobre cómo se refleja dicha realidad en los medios informativos impresos. La metodología seguida ha sido, en primer lugar, cualitativa, ya que se realizó una dinámica de grupo, y en segundo lugar cuantitativa a través de una encuesta telefónica a una muestra representativa de la población Este estudio nos ha permitido analizar cómo percibe la población de la CAPV la realidad tratada en el capítulo dos sobre la presencia de las mujeres en el mercado laboral, y tener el punto de vista de los y las lectoras sobre el análisis de prensa escrita que realizamos en los capítulos tres y cuatro, y de las publicaciones femeninas distribuidas junto con un diario analizadas en el capítulo cinco.

Las conclusiones de este capítulo suponen la elaboración analítica de los datos obtenidos en las encuestas, que van desde los hábitos de lectura de la población hasta el grado de conocimiento de la Ley de Igualdad. Se mencionan a continuación tan solo un par de ejemplos representativos de dichas conclusiones. Aunque el $90 \%$ de los encuestados afirman que en los últimos 10 años el número de directivas/empresarias ha aumentado, el 71,8\% no es capaz de citar el nombre de una directiva o empresaria. Y el $85,5 \%$ de la muestra afirma que aparecen menos mujeres directivas en prensa de las que verdaderamente hay en la realidad. 


\section{Voces representativas: hablan las protagonistas}

El último capítulo recoge los resultados de una jornada organizada por este equipo de investigación de la Universidad de Deusto en mayo de 2007, la cual logró efectivamente convertirse en un punto de encuentro, reflexión y debate en el espacio universitario sobre la presencia de las mujeres en el ámbito empresarial y su reflejo en los medios de comunicación. Esta actividad se dividió en dos sesiones. La primera, bajo el título de Mujeres, empresa y Ley de Igualdad, contó con la participación de Ana Aguirre, responsable de Formación de Emakunde, Carmen Arrazola, empresaria y directora de Proyectos, Elena Carantoña, socia de MB2, y Conchi Roussell, directora de CR Comunicación. La segunda sesión, Mujeres, Empresa y Medios de Comunicación contó con las aportaciones de Natividad Abril, periodista y profesora de la UPV-EHU, Charo Izquierdo, directora de Yo Dona, Amparo Moreno, catedrática de la Universidad Autónoma de Barcelona y Arantza Zugasti, directora adjunta de Noticias de Guipúzcoa. El interés que motivaron las ponencias de la jornada hizo que se prolongara el debate con una serie de preguntas y comentarios que dieron lugar a un fructífero intercambio de ideas. Todo ello nos ha llevado a presentar a continuación una selección de las ponencias presentadas por Carmen Arrazola, Elena Carantoña, Charo Izquierdo, Conchi Roussel y Arantzazu Zugasti con el fin de dar a conocer estas aportaciones de modo más amplio. Se ha decidido expresamente mantener el tono propio de la presentación para tratar de conservar de algún modo la cercanía del formato original. Como se puede apreciar, las presentaciones incluidas en esta selección ofrecen una gran variedad en cuanto al contenido, longitud o acercamiento a los temas propuestos, pero creemos que en esa diversidad reside la riqueza de sus aportaciones y por ello ofrecemos estas voces. En ciertas ocasiones hay formulaciones y modos de expresión que se adaptan más al estilo oral que al propio de la escritura, pero ello no es obstáculo para que la tarea de divulgación, de reflexión y de compromiso que transmiten llegue con fuerza en la lectura. En primer lugar, Carmen Arrazola nos recuerda que la economía es una ciencia social, y que un hecho económico implica a todo el resto de competencias, incluidas las 
emocionales, porque los procesos económicos están determinados por las personas, y su lógica, es la de las relaciones sociales. Por ello, el miedo, las frustraciones, las tensiones, las inquietudes, los deseos y las aspiraciones de las personas son determinantes en los acontecimientos económicos. Afirma que la economía es indisoluble con la cultura, con las formas de vida; por ello llegará un día en que la economía será una actividad de mujeres, como lo empiezan a demostrar las aulas de las universidades con presencia mayoritaria de mujeres. A partir de los datos extraídos de sus investigaciones, demuestra que las desigualdades existen en el mercado laboral y concluye que la tarea que debemos proponernos es la de superar el discurso, involucrar a los hombres y a las empresas en las medidas de igualdad como algo bueno para ellos y para las empresas. Elena Carantoña analiza en su presentación cómo se refleja el género en uno de los principales instrumentos de información corporativa de las empresas: las memorias de sostenibilidad o informes de responsabilidad social. Mediante la explicación del concepto de responsabilidad social investiga qué lugar ocupa en ella el género, tanto para los estándares internacionales que la promueven como en los propios informes que las empresas presentan, y a través de un ejemplo concreto, el de Telefónica de España, ilustra las posibilidades que tiene la responsabilidad social tanto para avanzar en la igualdad como para mejorar notablemente la información sobre la situación de la mujer en la empresa. Concluye su presentación con una propuesta de indicadores de género para la responsabilidad social, y afirma que el género forma parte del sistema de responsabilidad social, pero todavía no ha alcanzado los niveles de desarrollo que tienen la filantropía o el medioambiente. Queda mucho por hacer para clarificar conceptos y proporcionar a las empresas instrumentos útiles para mejorar la información. Pero esta es una evolución imparable y cualquier avance tiene repercusiones inmediatas en la vida de la empresa y de las mujeres profesionales. Charo Izquierdo, por su parte, analiza lo que son y han significado en España las llamadas revistas femeninas de moda y hace una descripción exhaustiva de la revista Yo Dona, que ella dirige, para acercarnos a la comprensión del significado de esta publicación en los parámetros de género en el panorama publicitario y editorial del Estado español. 
Conchi Roussel nos recuerda que la preparación profesional de la mujer ha mejorado mucho en las últimas décadas; sin embargo, esto no se refleja en cuanto a la incorporación de mujeres a los puestos directivos, que si bien es más alta en la Administración pública, no es así en la empresa privada. Muchas mujeres siguen partiendo de una mentalidad que concibe su salario como un complemento. A esto hay que añadir que, por tradición cultural, la mujer no ha sido entrenada en la ambición para asumir la responsabilidad de tener que mantener un hogar y una familia. En relación con los medios de comunicación, la autora ofrece un breve panorama de la situación actual y concluye con una serie de tareas pendientes para alcanzar una mayor justicia e igualdad en la transmisión de la información. Arantzazu Zugasti estudia la realidad de las mujeres en los medios de comunicación y de sus palabras se desprende que aún hay mucha segmentación por áreas de trabajo y que no hay mujeres en cargos de responsabilidad. Concluye que las mujeres aún no tenemos un discurso normalizado cuando ejercemos tareas de responsabilidad por lo que tendremos que seguir empujando porque socialmente ya ha desaparecido en el discurso público la necesidad de reivindicar el tema de la igualdad.

\section{Conclusiones}

El estudio pone de relieve dónde y cómo se encuentran las mujeres en la realidad empresarial e informativa en los espacios de poder económico y reivindicar la visibilidad de los logros de cada vez más mujeres, para así contribuir a paliar una situación de desequilibrio. El colectivo de mujeres empresarias y directivas va ir adquiriendo una mayor fuerza y presencia en el panorama nacional e importa resaltar sus logros en un entorno en el que en ocasiones pasa desapercibido entre las grandes cifras macroeconómicas y en una cultura empresarial y mediática que prima lo masculino. Si bien las mujeres ya tienen una presencia mayoritaria en las universidades, es necesario esperar algún tiempo para que su acceso a los puestos directivos sea 
habitual. Importa generar estrategias políticas y sociales definidas que ayuden a la superación del techo de cristal y favorezcan la conciliación de la vida laboral y familiar.

Como conclusión al libro en su totalidad, se advierte que los datos recogidos del análisis de prensa y de la percepción que ciudadanos y ciudadanas tienen del índice de participación de las mujeres en espacios de poder y ámbitos de decisión económica y empresarial revela que se han producido avances significativos, pero que el camino que queda por recorrer es aún muy largo. El poder y la autoridad vienen de la mano de la autonomía, y ésta se construye a través de la educación y de la libertad, pero también mediante la independencia económica. El poder político de muchas mujeres que aparecen en las conocidas listas de personas influyentes no ha redundado necesariamente en políticas más radicales de paridad ni en la visibilidad de las mujeres como sujetos legítimos y legitimados para compartir esos espacios de poder. A las bases de poder tradicionales todavía sustentadas en paradigmas masculinos se impone una realidad en constante transformación y más plural. Por tanto, se impone una nueva forma de liderazgo empresarial, más abierto, flexible y plural, para la que el papel de las mujeres es fundamental. Y los medios de comunicación han de ser conscientes de esta realidad y de la responsabilidad que tienen, como instituciones sociales, en esta lucha progresiva de hombres y mujeres por alcanzar unas cotas reales de igualdad. 


\section{BIBLIOGRAFÍA}

- Aierbe, Peio (2003), Análisis de prensa 2002. Inmigración, racismo y xenofobia, San Sebastián, Mugak.

- Barberá, Ester, Sarrió, Maite y Ramos, Amparo (coord) (2000): Mujeres directivas: promoción profesional en España y el Reino Unido. Exceptional Women: The Career Paths of Women Managers in Spain and the UK. Valencia, Universitat de Valencia.

- Barberá, Ester (dir.) Rompiendo el techo de cristal: los beneficios de la diversidad de género en los de dirección. http://www.uv.es/iued/investigacion/proyectos/resumen-teccho-cristal.pdf

- Casas, José Ignacio (1987), La participación laboral de la mujer en España, Madrid, Instituto de la Mujer.

- Chinchilla, Nuria (2000), «Mujer y empresa en el siglo XX», IESE, Revista de antiguos alumnos, septiembre, pp. 18-28.

- Chinchilla, Nuria, Poelmans, Steven y León, Consuelo (2005), Directivas en la empresa: criterios de decisión y valores femeninos en la empresa, Internacional Center of Work and Family.

- Corral Natalia, Antón, Fernando, Segura, Inmaculada, Filella, Miriam, Corredera, Julián, Escalonilla, Rafael, Von Schellwitz, Christian y Torres, Enrique (2005), «Conciliación vida laboral y vida personal y familiar», Manual de la empresa responsable, Madrid, Biblioteca Empresarial Cinco Días, cap. 9, pp. 219-236.

- Fernández De La Vega, María Teresa (2005), intervención de la vicepresidenta primera, ministra de la Presidencia y portavoz del Gobierno en la presentación del informe Diversidad de género en los consejos de administración de las sociedades españolas cotizadas y cajas de ahorros españolas (15 de noviembre) www.fef.es/z_contenido/Intervención_Vicepresidenta_acto_Diversidad_de_Genero_14 _11_05.pdf 
- García Añón, María (2007), «Evaluación de instrumentos que influyen en la difusión de noticias sobre la mujer», Actas del Foro Internacional Comunicación e Xénero, Pontevedra, pp. 343-374.

- Gómez Ansón, Silvia (2005), Diversidad de género en los consejos de administración de las sociedades cotizadas y cajas de ahorros españolas, Madrid, Fundación de Estudios Financieros.

- Grande, I. y Abascal, E. (2001), Fundamentos y técnicas de investigación comercial, Madrid, Esic.

- Gross, Liza (1995), La mujer como noticia y agente de cambio en la institución noticiosa, San José: SEM.

- Igartúa, J. José (2006), Métodos cuantitativos de investigación en comunicación, Barcelona, Editorial Bosch.

- Maruani, Margaret (2000), Trabajo y empleo de las mujeres, Madrid, Fundamentos.

- Mercadé, Anna (2007), Dirigir en femenino, Barcelona, Gestión 2000.

- Moreno Sardà, Amparo (1998), La mirada informativa, Barcelona, Bosch.

- Van Dijk, Teun A. (1980/1990), La noticia como discurso. Comprensión, estructura y producción de la información, Barcelona, Paidós Comunicación. . (1993/2003), Racismo y discurso de las élites, Barcelona, Gedisa. . (1997), Racismo y análisis crítico de los medios, Barcelona, Paidós.

- VVAA. (2006), El tratamiento de la violencia de género en la prensa vasca, Bilbao, Servicio Publicaciones Universidad de Deusto.

- VVAA. Dirigir en femenino (2009). Madrid, LID.

- Zikmund, W. G. y Aldás Manzano, J. (2003), Fundamentos de investigación de mercados, Madrid, Thomson. 